\title{
Sampling an Intrinsic Foot Muscle Improves Electrodiagnostic Sensitivity for Peripheral Neuropathy
}

\section{Jin Jun Luo*}

Departments of Neurology and Pharmacology, Lewis Katz School of Medicine at Temple University, Philadelphia, PA, USA

\section{Correspondence to:}

Jin Jun Luo, MD, PhD, FAAN, FAANEM

Professor of Neurology and Pharmacology

Lewis Katz School of Medicine at Temple

University, Philadelphia, Pennsylvania, USA

E-mail: jluo@temple.edu

Received: March 19, 2016

Accepted: June 15, 2016

Published: June 30, 2016

Citation: Luo JJ. 2016. Sampling an Intrinsic Foot Muscle Improves Electrodiagnostic Sensitivity for Peripheral Neuropathy. J Neurol Exp Neurosci 2(1): 12-14.

Copyright: (C) 2016 Luo. This is an Open Access article distributed under the terms of the Creative Commons Attribution 4.0 International License (CC-BY) (http://creativecommons.org/licenses/ by/4.0/) which permits commercial use, including reproduction, adaptation, and distribution of the article provided the original author and source are credited.

Published by United Scientific Group

\begin{abstract}
Routinely performed electrodiagnostic study may be normal in some subjects with mild peripheral neuropathy such as acral dysesthesia and/or weakness and, therefore, fails to confirm the diagnosis at the early stage. Aiming to improve electrodiagnostic sensitivity, we studied critical muscle sampling in electromyography. Subjects with clinical diagnosis of peripheral neuropathy and with normal conventional electrodiagnostic findings were initially recorded. An additional muscle sampling on the first dorsal interosseous pedis was performed. Based on the observation whether there was an absence or presence of abnormal electromyography findings in the muscle of first dorsal interosseous pedis, they were divided into normal group-A or abnormal Group-B. Relationships between the frequency of the abnormal electrodiagnostic study and comorbidities were analyzed. Group-A with 63 subjects (age $45.0 \pm$ 13.0 years old, Male/Female $=16 / 47$ ) while Group-B with 73 subjects (age 54.2 $\pm 13.5, \mathrm{M} / \mathrm{F}=29 / 43$ ) were identified based on whether there was the absence or presence of fibrillation potentials and positive sharp waves, an indicator of active denervation process, in the muscle of first dorsal interosseous pedis. More than half of the cohort had abnormal findings, which was increased in frequencies in diabetes, distal weakness, elderly and male populations. Sampling the first dorsal interosseous pedis improves the electrodiagnostic sensitivity for peripheral neuropathy.
\end{abstract}

\section{Keywords}

Active denervation, Electrodiagnostic study, Electromyography, Fibrillation potentials and positive sharps, First dorsal interosseous pedis, Neuropathy, Sustained activities

\section{Abbreviations}

EDx: Electrodiagnostic Study; EMG: Electromyography; F1-DIP: First Dorsal Interosseous Pedis; NCS: Nerve Conduction Study; PN: Peripheral Neuropathy

\section{Introduction}

Peripheral neuropathy $(\mathrm{PN})$ is a common neuromuscular disorder affecting approximately 20 million Americans, causing significant negative impact on the quality of life and creating an economic burden [1]. Electrodiagnostic study (EDx) such as nerve conduction (NCS) and needle electromyography (EMG) is a useful tool in confirming the diagnosis of PN [2]. However, it is frequently encountered that patients who present with typical but mild symptoms of PN with a stocking-glove-like pattern of sensory deficits and/or distal weakness in 
the limbs may have normal findings in conventional EDx. Therefore, they may then get delayed in diagnosed and receiving appropriate medical attention. Those who had a normal NCS/EMG study may have undergone a suboptimal EDx evaluation for their "mild" neuropathies. A recent study by Siddiqi and colleagues suggested that sampling the fourth dorsal interosseous pedis (F4-DIP) muscle may be a useful muscle to include in routine electrodiagnostic evaluation [3]. In this report, we present our clinical observational findings that an additional distal muscle sampling on the first dorsal interosseous pedis (F1-DIP) in EMG would improve the EDx sensitivity for $\mathrm{PN}$.

\section{Methods}

An EMG laboratory database and clinical charts were retrospectively reviewed. Patients who had been referred to the EDx laboratory for $\mathrm{PN}$ were recorded. The routine conventional EDx for PN included NCS and needle EMG. According to our EDx laboratory protocol for evaluating peripheral neuropathy, NCS, including F-waves and H-reflexes, was performed on motor nerves of median, ulnar, fibular (formerly called peroneal), tibial, and sensory nerves of median, ulnar, radial and sural sensory nerves in at least one arm and one leg. EMG using a concentric electrode was performed on muscles of, at least, deltoid, biceps, triceps, first dorsal interosseous, and abductor pollicis brevis in one arm; and medial vastus, tibialis anterior, medial gastrocnemius, tibialis posterior in one leg. Data of EMG including insertional, spontaneous, and volitional activities; configuration of motor unit potentials; and recruitment patterns were collected [4]. Insertion-triggered or spontaneous fibrillation potentials and positive sharp waves were defined as a rhythmic or semi-rhythmic discharge pattern (e.g. less than 5 milliseconds in duration and biphasic or triphasic waveforms with initial positivity in morphology for fibrillations) of spontaneous depolarization of a single muscle fiber at rest. Additionally, an intrinsic foot muscle, the first dorsal interosseous pedis (F1-DIP), was sampled by inserting a concentric EMG needle obliquely at an angle of about $30^{\circ}$ immediately proximal to the first and second metatarsal heads. F1-DIP is an easily accessible muscle and less prone to blunt trauma as compared with the other foot muscles. Patients tolerated well. The skin temperature was monitored and maintained at $32{ }^{\circ} \mathrm{C}$ or above for the upper and $30{ }^{\circ} \mathrm{C}$ or above for the lower extremities during the EDx evaluations. Nicolet Biomedical EMG machine (Viking Select, version 10, Madison, WI) was employed.

The clinical diagnosis of PN was made based on the clinical information, which was defined as the clinical findings from history, clinical manifestation, and neurologic examination, such as presentation of symptoms of numbness and tingling with or without weakness in distal limbs, and signs of sensory deficits such as light touch, temperature or vibration sensations, and decrease or absence of ankle reflex [5].

Subjects with an EDx diagnosis, neuroimaging or history of radiculopathy, entrapment syndrome, traumatic nerve injury, and myopathy or myositis were excluded. Subjects with normal conventional EDx findings and with additional F1-
DIP sampling in EMG were collected. They were divided into group-A (normal) or Group-B (abnormal) based on the absence or presence of EMG findings in F1-DIP.

The relationships between the frequency of abnormal EDx in F1-DIP and the presenting symptoms or concomitant medical conditions were analyzed. Chi-square, Z-test and t-test for 2 independent means of two-tailed analysis were performed and $\mathrm{p}<0.05$ was considered statistically significant.

\section{Results}

One hundred and thirty-six subjects (age: $49.9 \pm 14.0$ years old, mean \pm SD, Male/Female $=46 / 90)$ who met the inclusion criteria were studied. Of these subjects, 73 (53.7\%) (age $54.2 \pm 13.5$ y.o., $\mathrm{M} / \mathrm{F}=29 / 43$ ) were found to have abnormal EMG findings in F1-DIP showing fibrillation potentials and positive sharp waves (Group-B) indicating an ongoing active denervation, while $63(46.3 \%, 45.0 \pm 13.0$ y.o., $M / F=16 / 47$ ) were normal (Group-A). A significant difference $(p<0.05)$ was observed between these two groups in age $(p=0.001)$, weakness $(p=0.03$, as the frequency of presenting symptoms), and presence of diabetes ( $p=0.001$, as the comorbidity). Notably, weaknesses in clinical presentation were subjective (5/5 on MRC), or very mild if any $\left(5^{-/ 5}\right)$. Interestingly, an increased rate of male gender was observed in Group-B ( $\mathrm{p}=0.03)$ although females remained predominant (Table 1). No significant differences were seen in symptoms of dysesthesia and back pain between these two groups. Due to smaller numbers, symptoms of cramping and chronic medical conditions such as hepatitis, human immunodeficiency virus infection, chronic kidney disease, post status of having received chemotherapy were not analyzed.

\section{Discussion}

On this small scale of observational study, we addressed whether an additional distal muscle sampling of F1-DIP in EMG would improve EDx sensitivity for the diagnosis of PN. Our results support the previous notion by Siddiqi and colleagues that sampling a distal intrinsic foot muscle may be a useful muscle in routine EDx evaluation [3] and extend to that sampling F1-DIP may improve the EDx sensitivity for $\mathrm{PN}$ at the early stage.

F1-DIP was chosen because it is one of the most remote muscles in the body innervated by neurons from the spinal cord and more prone to early involvement in $\mathrm{PN}$ intuitively due to the dying back phenomenon $[6,7]$. Several intrinsic foot muscles can be chosen in this regard but sampling FDIP has advantages. Firstly, it is less likely subjected to a traumatic injury at its anatomical location, therefore, the findings are less likely influenced by a local injury. Secondly, it is easily approached. Thirdly, it is less painful than sampling other intrinsic foot muscles, such as abductor digiti minimi pedis or abductor halluces, and therefore it is well tolerable by patients. Fourthly, there is no risk in adversely injuring adjacent structures such as large blood vessels or a nerve trunk. It is well known that abnormal activities such as fibrillation potentials and positive sharps can be seen in health subjects in extensor 
digitorum brevis, abductor digiti quinti pedis and abductor hallucis muscles simply due to their anatomic locations which are prone to mechanically traumatic injury [8-10]. However, argument exists against this notion stating that those abnormal activities never occur alone and their occurrence is pathologic [11]. Siddiqi and colleagues demonstrated that a low incidence of fibrillation potentials was seen in F4-DIP muscle and the presence of fibrillation potentials in F4-DIP correlates well with the overall neurologic condition [3] which was similarly seen in our study with F1-DIP.

Notably, there is no defined recommendation on sampling muscles in EMG evaluation as to make the diagnosis of PN nor addressing for the EDx sensitivity in documenting $\mathrm{PN}$ in the AANEM guideline [4]. The results from the current study showed that an additional distal muscle sampling of F1-DIP improved EDx sensitivity by disclosing abnormalities in 53.7\% subjects with clinically suspected PN who initially failed to show abnormality in the conventional EDx studies (Table 1). In other words, sampling the intrinsic foot muscle F1-DIP in $\mathrm{EMG}$ may be impressive in disclosing EDx evidence of an ongoing distally active denervation in patients with a mild PN. Approximately half of our patients who were initially "normal" in the conventional EDx without sampling F1-DIP eventually displayed "abnormal" by an additionally sampling F1-DIP (Table 1). Findings of the current observation were in agreement with those of distal axonopathy in a pattern of length-dependent or dying back described in pathology, which mostly affected distal, especially in the lower limbs [6, 7]. A study designing to address its sensitivity and specificity is ongoing. Analysis of comorbidity showed that diabetes mellitus, an established risk factor for PN, predisposed the appearance of abnormal EMG findings in F1-DIP ( $\mathrm{p}=$ 0.001 ) which is supported by literature [7]. Notably, motor

Table 1: Demographic, clinical and electrodiagnostic data.

\begin{tabular}{|c|c|c|c|}
\hline & Group-A & Group-B & Total \\
\hline \multicolumn{4}{|l|}{ Subjects } \\
\hline $\mathrm{N}$ & $63(46.3 \%)$ & $73(53.7 \%)$ & 136 \\
\hline Age (years)* & $45.0 \pm 13.0$ & $54.2 \pm 13.5$ & $49.9 \pm 14.0$ \\
\hline Male/female ${ }^{\#}$ & $16 / 47(34 \%)$ & $29 / 43(67 \%)$ & $46 / 90(51 \%)$ \\
\hline \multicolumn{4}{|c|}{ Presenting symptoms } \\
\hline Dysesthesia & 38 & 41 & 79 \\
\hline Back pain & 21 & 26 & 47 \\
\hline Weakness $^{\#}$ & 3 & 11 & 14 \\
\hline Cramping & 3 & 1 & 4 \\
\hline Others & 1 & 3 & 4 \\
\hline \multicolumn{4}{|l|}{ Comorbidity } \\
\hline $\mathrm{DM}^{*}$ & 3 & 10 & 13 \\
\hline Hepatitis/HIV & 1 & 3 & 4 \\
\hline CKD & 0 & 1 & 1 \\
\hline Chemotherapy & 1 & 0 & 1 \\
\hline Others & 3 & 2 & 5 \\
\hline
\end{tabular}

Group-A: Normal F1-DIP; Group-B: Abnormal F1-DIP with fibrillation potentials or sharps.

DM: diabetes mellitus; CKD: chronic kidney disease.

$*$ : $\mathrm{p}=0.001, \#: \mathrm{p}=0.03$ as compared between Group-A and Group-B. deficits with distal weakness, even though mostly subjective or very mild, correlated with the emergence of abnormal EMG findings in F1-DIP ( $p=0.03$ ). Therefore, patients with PN at the early stage who had normal findings in a conventional EDx may be delayed in diagnosis and receiving appropriate medical attention.

Interestingly, aging seemed to be another risk factor predisposing to the development of abnormal EMG findings $(p=0.001)$. Our observations also suggested an increased trend of risk in men to yield active denervation in F1-DIP ( $p=0.03)$, which is in agreement with literature [8], although females with PN remained predominant (Table 1). However, our observation may need to be validated through a larger scale study. Sensory symptoms such as dysesthesia and back pain may not be the factors prone to generating EDx abnormality in F1-DIP in our studies.

In summary, sampling intrinsic foot muscle improves the EDx sensitivity for PN, particularly in subjects with diabetes, distal weakness, elderly, and possibly males.

\section{Acknowledgments}

The author is grateful to Favio Bumanlag, MB, MS, for technical support and to Jie Feng, $\mathrm{PhD}$, for statistical advice.

\section{References}

1. Congress USA ST. Senate Report, Appropriation Bill 2005. In: Departments of Labor HaHS, and Education, and Related Agencies editor. 108-135.

2. Misra UK, Kalita J, Nair PP. 2008. Diagnostic approach to peripheral neuropathy. Ann Indian Acad Neurol 11(2): 89-97. doi: 10.4103/09722327.41875

3. Siddiqi ZA, Nasir A, Ahmed SN. 2007. The fourth dorsal interosseus pedis muscle: a useful muscle in routine electromyography. $J$ Clin Neurophysiol 24(6): 444-449. doi: 10.1097/WNP.0b013e31815c4605

4. Jablecki CK, Busis NA, Brandstater MA, Krivickas LS, Miller RG, et al. 2005. Reporting the results of needle EMG and nerve conduction studies: an educational report. Muscle Nerve 32(5): 682-685. doi: 10.1002/mus.20422

5. Luo JJ, Sivaraaman K, Nouh A, Dun NJ. 2014. Elevated plasma level of homocysteine is an independent risk factor for peripheral neuropathy. British J Med Med Res 4(1): 161-169. doi: 10.9734/BJMMR/2014/5206

6. Schaumburg HH, Spencer PS. 1979. Clinical and experimental studies of distal axonopathy-a frequent form of brain and nerve damage produced by environmental chemical hazards. NYAS 329: 14-29. doi: 10.1111/j.1749-6632.1979.tb15331.x

7. Chung T, Prasad K, Lloyd TE. 2014. Peripheral neuropathy: clinical and electrophysiological considerations. Neuroimaging Clin NAm 24(1): 49-65. doi: 10.1016/j.nic.2013.03.023.

8. Boon AJ, Harper CM. 2003. Needle EMG of abductor hallucis and peroneus tertius in normal subjects. Muscle Nerve 27(6): 752-756. doi: 10.1002/mus.10356

9. Falck B, Alaranta H. 1983. Fibrillation potentials, positive sharp waves and fasciculation in the intrinsic muscles of the foot in healthy subjects. $J$ Neurol Neurosurg Psychiatry 46(7): 681-683. doi: 10.1136/jnnp.46.7.681

10. Morgenlander JC, Sanders DB. 1994. Spontaneous EMG activity in the extensor digitorum brevis and abductor hallucis muscles in normal subjects. Muscle Nerve 17(11):1346-1347. doi: 10.1002/mus.880171115

11. Menkes DL, Sander HW. 2000. Plantar muscle fibrillations and positive sharp waves. Muscle Nerve 23(6): 989-990. doi: 10.1002/(SICI)10974598(200006)23:6<989::AID-MUS25>3.0.CO;2-S 